\title{
Microwave Imaging Using CMOS Integrated Circuits with Rotating $4 \times 4$ Antenna Array on a Breast Phantom
}

\author{
Hang Song, ${ }^{1}$ Afreen Azhari, ${ }^{1}$ Xia Xiao, ${ }^{2}$ Eiji Suematsu, ${ }^{3}$ \\ Hiromasa Watanabe, ${ }^{4}$ and Takamaro Kikkawa ${ }^{1}$ \\ ${ }^{1}$ Research Institute for Nanodevice and Bio Systems, Hiroshima University, 1-4-2 Kagamiyama, Higashihiroshima, \\ Hiroshima 739-8527, Japan \\ ${ }^{2}$ School of Electronic and Information Engineering, Tianjin University, Tianjin 300072, China \\ ${ }^{3}$ Sharp Corporation, Fukuyama, Hiroshima 721-8522, Japan \\ ${ }^{4}$ Sharp Takaya Electronic Industry Co., Ltd., Yakage, Okayama 714-1211, Japan \\ Correspondence should be addressed to Hang Song; hangsong90@hiroshima-u.ac.jp
}

Received 22 December 2016; Accepted 28 March 2017; Published 24 April 2017

Academic Editor: Ahmed Toaha Mobashsher

Copyright (C) 2017 Hang Song et al. This is an open access article distributed under the Creative Commons Attribution License, which permits unrestricted use, distribution, and reproduction in any medium, provided the original work is properly cited.

A digital breast cancer detection system using $65 \mathrm{~nm}$ technology complementary metal oxide semiconductor (CMOS) integrated circuits with rotating $4 \times 4$ antenna array is presented. Gaussian monocycle pulses are generated by CMOS logic circuits and transmitted by a $4 \times 4$ matrix antenna array via two CMOS single-pole-eight-throw (SP8T) switching matrices. Radar signals are received and converted to digital signals by CMOS equivalent time sampling circuits. By rotating the $4 \times 4$ antenna array, the reference signal is obtained by averaging the waveforms from various positions to extract the breast phantom target response. A signal alignment algorithm is proposed to compensate the phase shift of the signals caused by the system jitter. After extracting the scattered signal from the target, a bandpass filter is applied to reduce the noise caused by imperfect subtraction between original and the reference signals. The confocal imaging algorithm for rotating antennas is utilized to reconstruct the breast image. A $1 \mathrm{~cm}^{3}$ bacon block as a cancer phantom target in a rubber substrate as a breast fat phantom can be detected with reduced artifacts.

\section{Introduction}

The research on breast cancer detection and breast monitoring using microwave has been studied widely in the past two decades. X-ray mammography has been used as a detection technology. However, it causes ionizing radiation. The microwave imaging is based on the facts that the normal breast tissue and the cancer tissue have contrast in electrical properties [1-3]. According to the frequency ranges, two kinds of methods are studied for breast imaging: tomography which utilizes the single frequency and time-domain confocal imaging which utilizes ultrawideband signals. The tomography aims at reconstructing the distribution of electrical properties in the whole breast by use of inversion method [4-8]. Meanwhile, the confocal imaging aims at indicating the position of the target by use of the delay time of the reflected signal [9-11]. Both methods have their own merits and demerits. The tomography can recover the entire information of the breast under test. However, it sometimes takes a long time and large calculation consumption to get the final results. It also encounters ill-positioned cases which cannot make the results convergent. On the other hand, as confocal imaging only employs the time delay information to estimate the position of the target, the data processing is relatively simpler than tomography. However, the complete information of the breast cannot be recovered.

Research groups around the world are developing prototype experiment systems based on either tomography or confocal imaging. Epstein et al. have developed a prototype tomography system using monopole antenna array [12, 13]. For the time-domain method, Klemm et al. [14,15] and Porter et al. [16-18] have developed the multistatic radar system, respectively. Fear et al. have developed a monostatic radar 


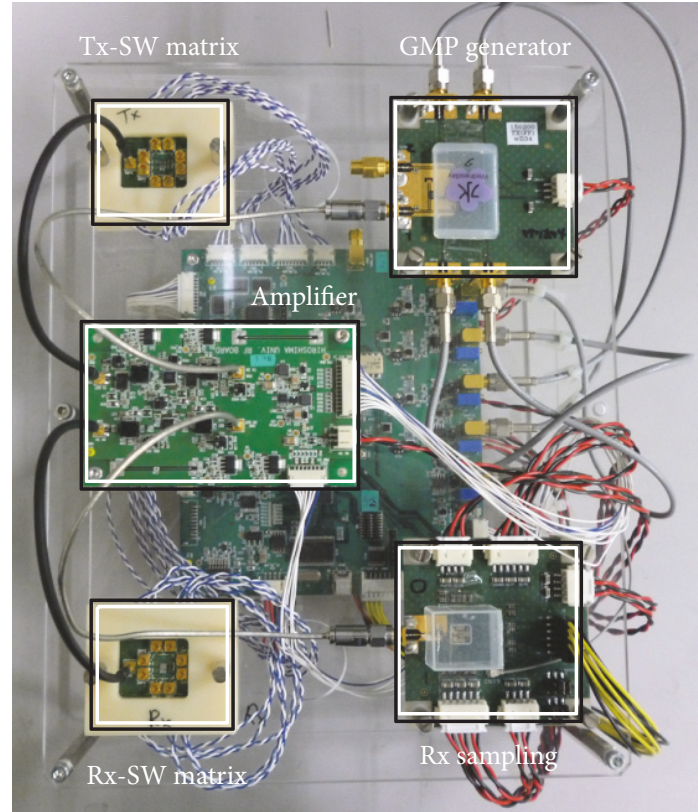

(a)

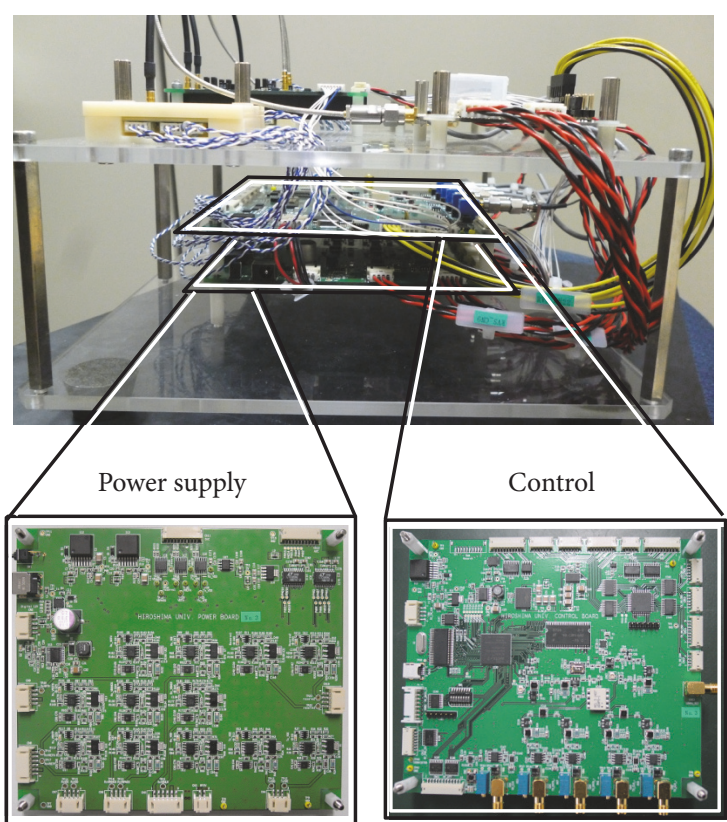

(b)

Figure 1: Photographs of the hardware system. (a) GMP generator, amplifier board, Tx switching matrix, Rx switching matrix, and Rx sampling circuits. (b) Power supply board and control board.

system $[19,20]$. Their systems have been tested on clinical cases and obtained some promising results. Nonetheless, these systems consist of large off-the-shelf equipment such as vector network analyzers (VNA), which make the system only be installed in the hospital and cannot be used for frequent examination at home. Therefore, miniaturization of the imaging system is essential for widespread use [21-23]. In our previous work, functional CMOS integrated circuits were developed for Gaussian monocycle pulse generation, highspeed sampling, and channel switching [24-30]. Additionally, a complete imaging system using these CMOS circuits was proposed and the feasibility was examined [31]. In this paper, the more compact digital detection system with rotating 4 $\times 4$ antenna array for higher resolution of both amplitude and time is presented and applied to a breast phantom. The rotating method is used for extracting the target reflection from the received signals. A signal alignment method based on the least square is proposed to eliminate the phase shifts caused by the system jitter. The bandpass filter is employed to mitigate the high-frequency noise introduced in the target reflection. The improvement of the imaging performance demonstrates the effectiveness of the signal processing methods.

This paper is organized as follows. Section 2 briefly describes the composition of the compact hardware system. Section 3 describes the breast phantoms and experiment settings and details the experiment operating procedures. Section 4 shows the data obtained from the system and specifies the signal processing methods. The effects of these methods on the imaging quality are discussed. In the final section, a conclusion is made.

\section{Hardware System Description}

Photographs of this system are shown in Figures 1(a) and 1(b). In this system, Gaussian monocycle pulse (GMP) trains which have the pulse width of $160 \mathrm{ps}$, the center frequency of $6 \mathrm{GHz}$, and the repetition rate of $100 \mathrm{MHz}$ is generated by a transmitter-chip (Tx-chip) $[29,30]$ and transmitted by a Tx module as shown in Figure 2. Figures 2(a) and 2(b) show Tx-chip wire bonding and a top view of Tx module, respectively. The Tx-chip size is $1.57 \mathrm{~mm} \times 1.06 \mathrm{~mm}$. The differential output pads for the GMP having the bandwidth of $7 \mathrm{GHz}(3 \mathrm{GHz}-10 \mathrm{GHz})$ of the Tx-chip are connected to GSG pads of the Tx FR-4 printed circuit board (PCB) by wire bonding. In order to shorten the length of the bonding wire, the surface of the Tx-chip and that of the PCB are aligned by countersinking the Tx-chip in the PCB. The resulting wire length is $680 \mu \mathrm{m}$.

Received signals are converted to digital data by a receiver-chip (Rx-chip). The equivalent sampling speed of the $\mathrm{Rx}$-chip is $102.4 \mathrm{GS} / \mathrm{s}$ [27]. The Rx-chip is assembled on FR4 PCB by wire bonding. The surface of the Rx-chip and that of the PCB are aligned to shorten the length of the bonding wire as shown in Figure 3. Figures 3(a) and 3(b) show Rx-chip wire bonding and a top view of $\mathrm{Rx}$ module, respectively. The $\mathrm{Rx}$-chip size is $2.3 \mathrm{~mm} \times 1.6 \mathrm{~mm}$.

The ultrawideband (UWB) GMP signal is distributed to a Tx-antenna of $4 \times 4$ matrix antennas [32] by a CMOS single-pole-eight-throw (SP8T) switching (SW) matrix [26]. In order to get rid of parasitic capacitances and inductances of the wire bonding, the flip-chip technology is used as shown in Figure 4. Figures 4(a) and 4(b) show SP8T flip-chip bonding 


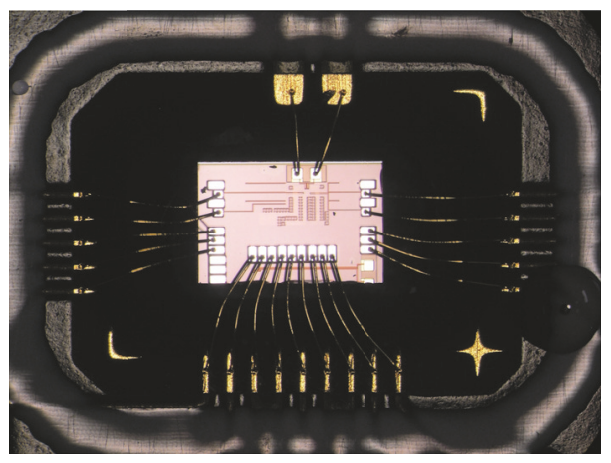

(a)

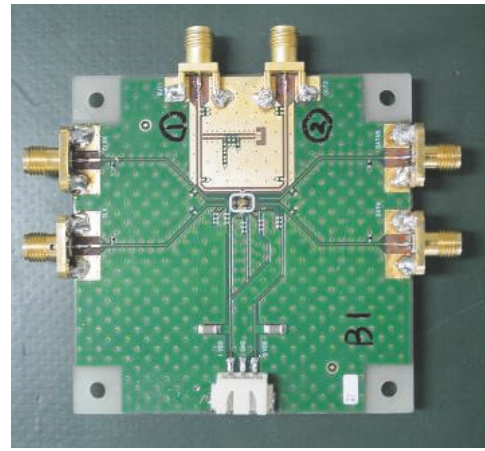

(b)

Figure 2: Photographs of the Tx-chip. (a) Wire bonding. (b) Top view of the Tx module.

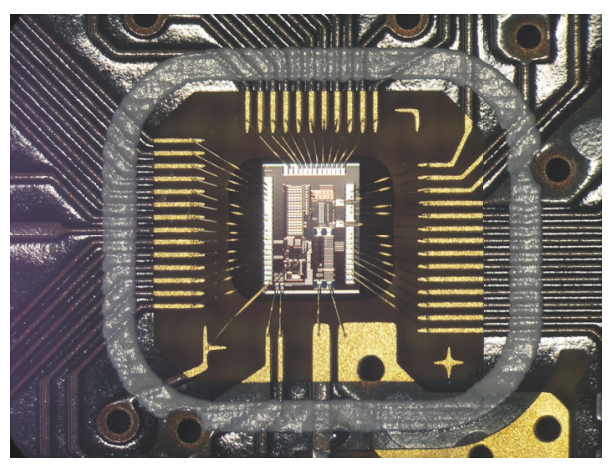

(a)

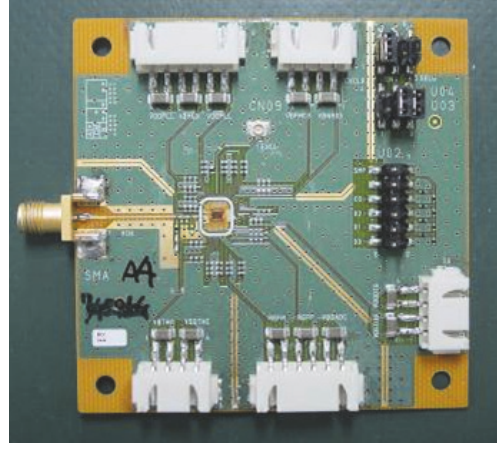

(b)

FIgUre 3: Photographs of the Rx-chip. (a) Wire bonding. (b) Top view of the Rx module.

and a top view of SW-module, respectively. The SW-chip size is $2.3 \mathrm{~mm} \times 1.6 \mathrm{~mm}$.

\section{Experiment}

The amplified GMP signal train is shown in Figure 5. A software is developed to control the SW matrix through the control board. The reflected signals are received by the receiving $(\mathrm{Rx})$ antennas in turn. The received signals are amplified by a LNA part on the amplifier board and subsequently digitalized by the sampling circuits. As the jitter exists in the system, the sampling procedure is repeated for 16 times to improve the resolution of the signal by averaging. As a result, the 4-bit ADC becomes an equivalent 8-bit ADC and 256 least significant bits (LSB) can be achieved. At last, the data are saved in the laptop computer for the offline postprocessing. Figure 6 shows the signal before and after averaging. The averaged signals are defined as the original signals in the postprocessing.

Experiments are conducted by rotating the $4 \times 4$ matrix antennas and changing detection positions for extracting the target reflection. Since there are jitters existing in the system, the signals obtained in different positions may have offsets in the time dimensions. An alignment method based on the least squared error is proposed to eliminate the phase shifts. The bandpass filter is applied to the target signal to suppress the noise. The confocal image is generated to demonstrate the effect of the signal processing method and the feasibility of the system.

The experimental setup is shown in Figure 7. The breast phantom is fabricated by use of a rubber since the rubber has similar dielectric properties to those of breast fat tissues. The breast phantom consists of two $2 \mathrm{~cm}$ thick rubber substrates. The size of the lower one is $15 \mathrm{~cm} \times 15 \mathrm{~cm}$ and the antenna array is consolidated on it. The center of the substrate is consistent with that of the antenna array. 16 cables are used to connect the $4 \times 4$ antenna array and SW matrix during the experiment [32]. The cables are confined by a plate with 16 small holes to reduce the movement of the cables because the connecting status may cause the signals to change a lot. The upper rubber substrate is cut and polished as a thin cylinder. The diameter of the cylinder is $130 \mathrm{~mm}$. A thin plate with a $130 \mathrm{~mm}$ diameter hole is fixed on the lower substrate, in which the cylinder rubber can be inserted and rotated. The centers of the hole and the cylinder substrate coincide with the ones of the lower substrate and the antenna array. The angular scale labels are printed on the plate. Therefore, the rotation experiment can be conducted accurately and the relative positions between antennas and phantom can be calculated.

A $1 \mathrm{~cm}^{3}$ bacon is utilized as the target to mimic the tumor since bacon has similar permittivity to the breast tumor. 


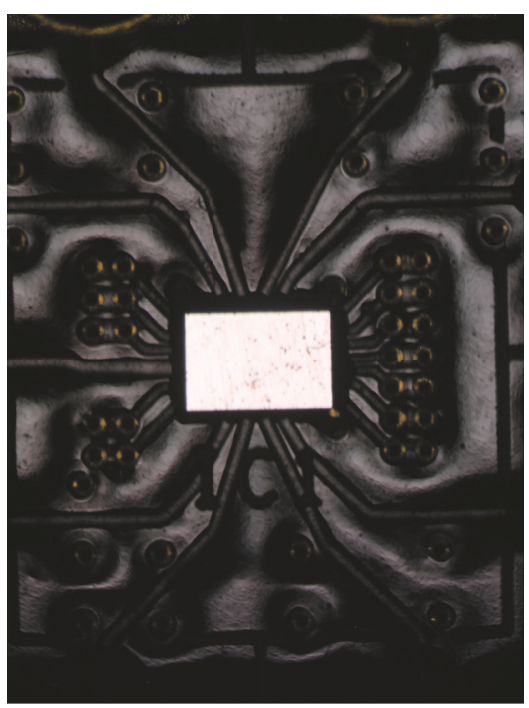

(a)

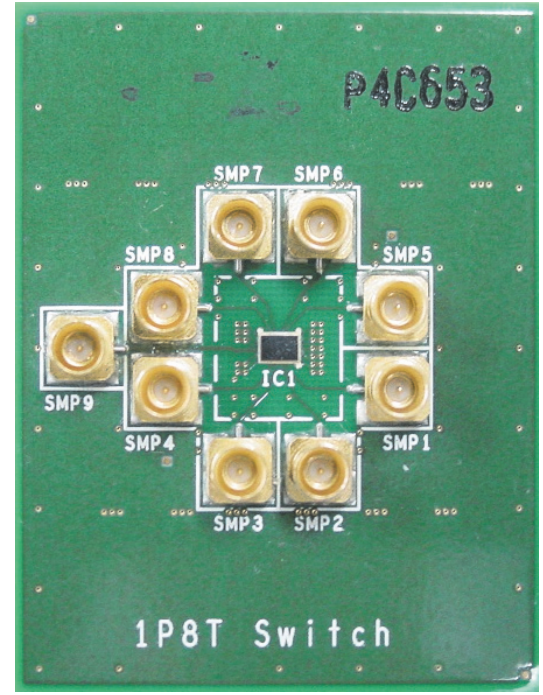

(b)

FIGURE 4: Photographs of the switching (SW) matrix chip. (a) Flip-chip bonding. (b) Top view of the SW matrix module.

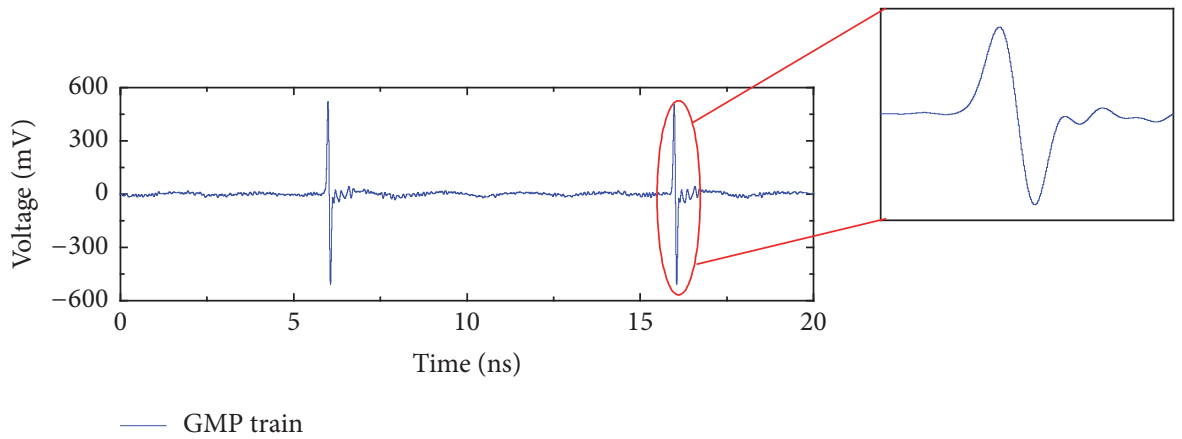

FIGURE 5: The Gaussian monocycle pulse train with the pulse width of $160 \mathrm{ps}$ and the repetition period of $10 \mathrm{~ns}$.

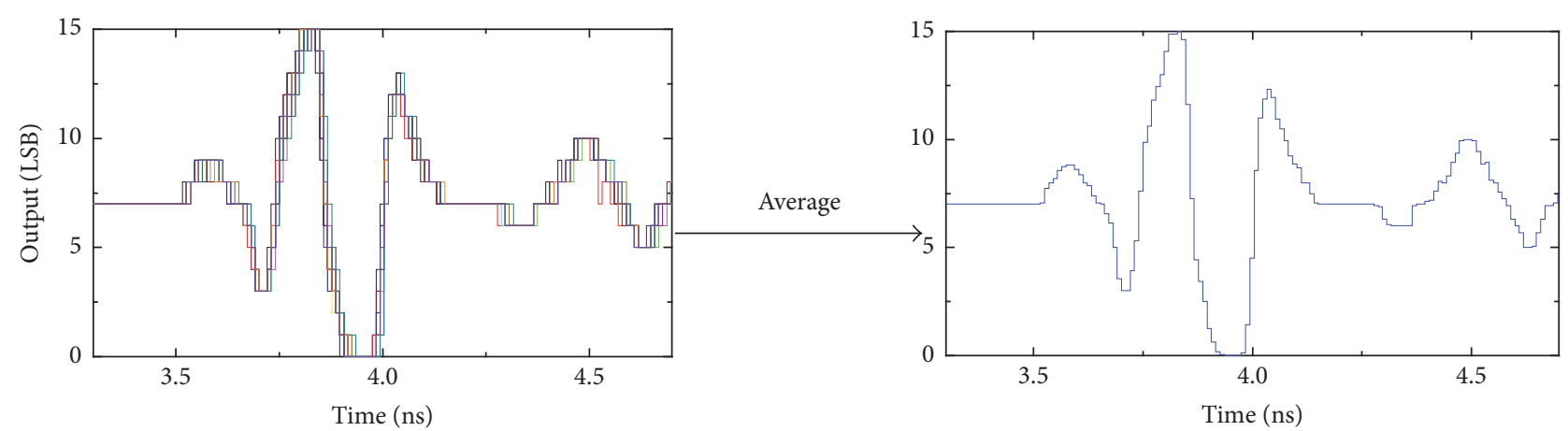

FIgURE 6: The raw 4-bit signal sampled serially in 16 times and the corresponding averaged one.

The electrical properties of phantom materials and breast tissues are shown in Figure 8.

A $10 \mathrm{~mm}$ depth hole is drilled into the upper cylinder rubber for inserting the target. The hole center is $15 \mathrm{~mm}$ from the center of the antenna center in $y$ direction. Place the cylinder into hollowed plate with angle scale. Since the thickness of the lower rubber is $20 \mathrm{~mm}$ and target size in $z$ direction is $10 \mathrm{~mm}$, the distance between the antenna array and the target center in $z$ direction is $25 \mathrm{~mm}$. The experiment is repeated by rotating the cylinder rubber from 0 to 360 degrees and the rotation step can be changed from 1 90 degrees. In this experiment, the rotation step is chosen as 10 degrees. Then, the experiment is carried out for 36 times. Figure 9 shows the schematic diagram of the experiment. 


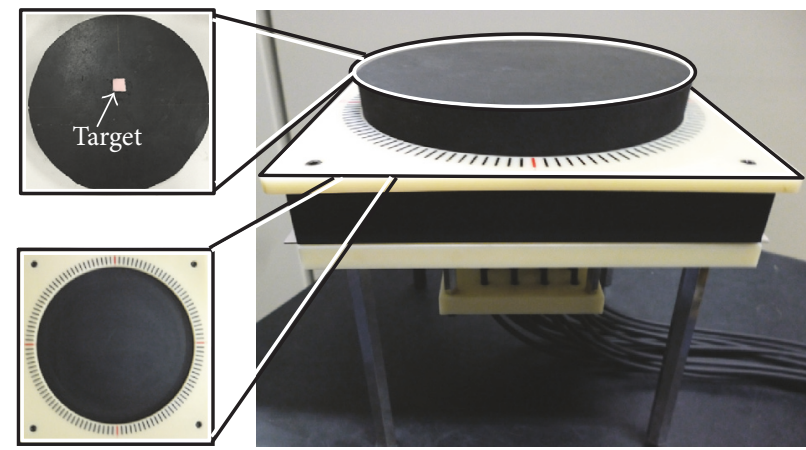

FIGURE 7: The breast phantom and the experimental setup.

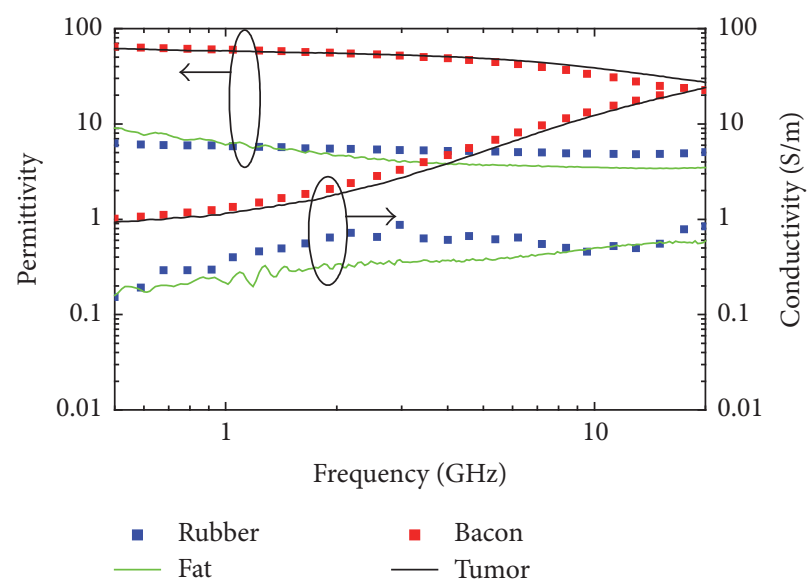

FIGURE 8: The electrical properties of phantom materials and breast tissues.

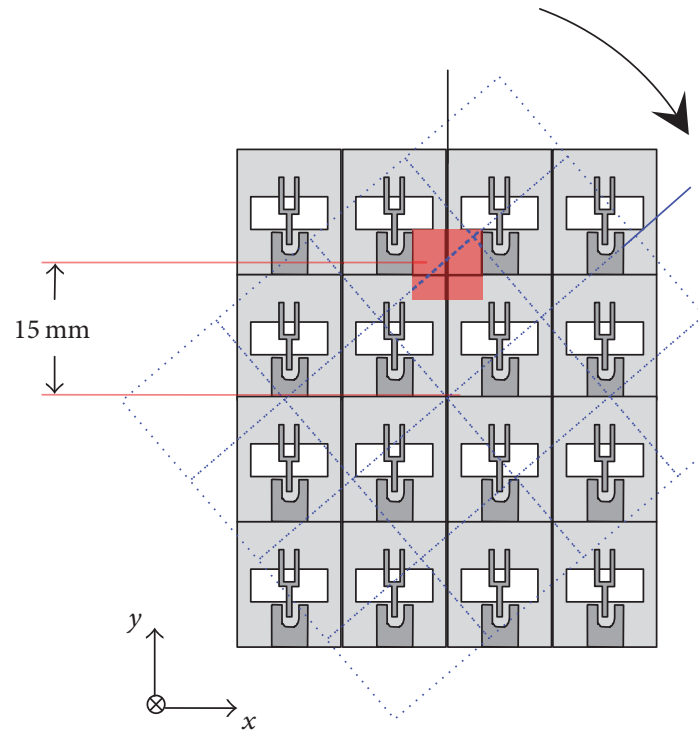

FIGURE 9: The change of relative positions between array and target in rotation experiment system.

During the rotation process, the positions between antennas are not changed. Therefore, the direct waves should be similar. However, the relative location of the target to the antenna array is varied at different angles. Therefore, the delay times of the target reflection are diverse. By averaging the signals at different angles, the direct wave remained. Meanwhile, the target reflection is reduced since the target reflections appear incoherently. The averaged signal is regarded as the reference signal and subtracted from the original ones so that the target reflection can be extracted. Utilize the delay 


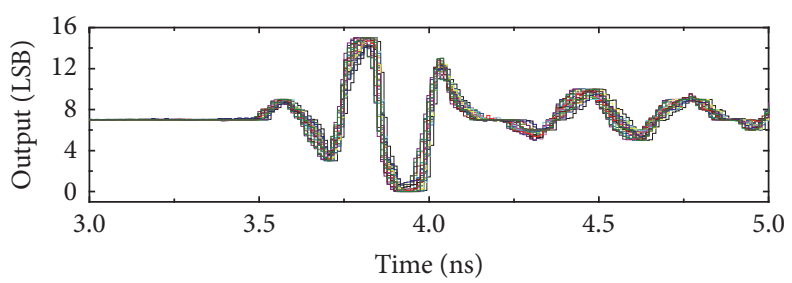

FIGURE 10: The original signals at 36 different angles from antenna pair Tx3-Rx7.

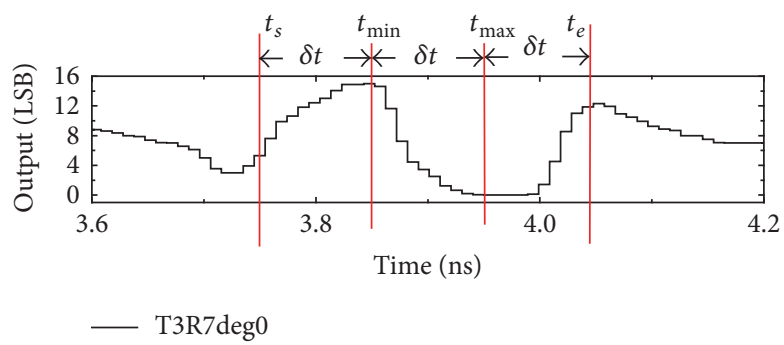

FIGURE 11: The time range for signal phase shift compensation.

time information of the target reflection, and the position of the target can be estimated. The following section will discuss the processing procedures to get the final reconstructed breast image to show the target position.

\section{Data Processing and Imaging}

After the experiment is completed, the data have been saved in the computer. The first step to process the data is averaging the 16-time raw data to generate the original signals. The experiment is conducted 36 times by rotating the detection system by 10 degrees so that 36 sets of original signals are processed. In order to evaluate the proposed rotation method, a set of signals when there is no target existing are also acquired. Define this set of signals as without signals. The purpose of the rotation experiment is to extract the target reflection by use of the signals at different angles. Figure 10 shows the original data from $\mathrm{Tx} 3-\mathrm{Rx} 7$ antenna pair.

Since the signals are from the same antenna pair, the shape of the waveform is almost the same. However, the signals have offsets in the time dimension because of the system jitter. Therefore, before averaging the signals, phase shift compensation is necessary to eliminate the jitter effect. The procedure of the compensation is as follows. First, choose the signals which are acquired at 0 degrees as the baseline. Then, adjust the corresponding signals in other sets to align to the baseline counterpart. The alignment process is to find a best phase shift factor to make the squared error between two signals be the minimum. This least square optimization problem can be defined as follows:

$$
\min _{\text {offset }} \sum_{n=t_{s}}^{t_{e}} \mid S_{0}\left(t_{n}\right)-\left.S_{m}\left(t_{n}+\text { offset }\right)\right|^{2}
$$

where $S_{0}$ is the baseline signal and $S_{m}$ is the signal from other sets. $t_{s}$ and $t_{e}$ are the start time and end time of the range for summation as shown in Figure 11. They are determined by the max-peak $\left(t_{\max }\right)$ and min-peak $\left(t_{\min }\right)$ of the baseline signal. The range $\left(t_{s}, t_{e}\right)$ is three times the interval between $t_{\max }$ and $t_{\text {min }}$.

After the compensation processing, the signals at different angles are aligned. Figure 12 shows the signal at 120 degrees compared with baseline signal before and after compensation. After compensation, the direct wave parts match well and the later reflection parts are different. This is because the distance between the target and the antenna array varies and the timing of the target reflection changes at different angles. The aligned signals are averaged to generate a reference signal as shown in Figure 13. By subtracting the reference signal from the original signals, the target response can be extracted as shown in Figure 14. The parts in the pink frame are the target reflection. Figure 14(a) shows the original waveform for $\mathrm{Tx} 3-\mathrm{Rx} 7$ antenna pair at $0^{\circ}$ rotation (red) and the corresponding reference waveform (blue). Differential waveforms are shown as diff (black). The differential waveforms for the rotation angles of $60^{\circ}, 90^{\circ}, 120^{\circ}$, $160^{\circ}, 180^{\circ}, 250^{\circ}, 300^{\circ}$, and $330^{\circ}$ are shown in Figures $14(\mathrm{~b}) \sim$ 14(i), respectively. As can be seen, the waveforms of the target reflection appear at different delay times.

In order to confirm the validity of the extracted target response using the reference signal method, the ideal artificial removal method is applied by subtracting the without signal set. Figure 15 shows the differential signals using the without signal before and after alignment, together with the signal using the reference signal.

Comparing the signals in Figures 15(a) and 15(b), it is obvious that the phase shift compensation is necessary because a small time shift will cause a large residues in direct wave range. Comparing the signals in Figures 15(b) and 15(c), 

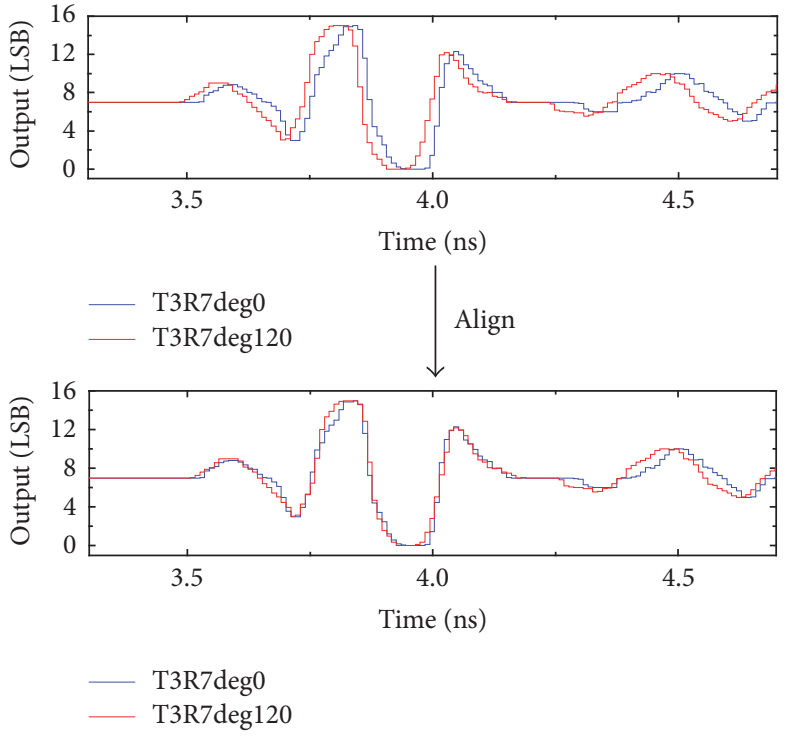

FIGURE 12: The comparison of baseline signal and the signal at 120 degrees before and after phase shift compensation.

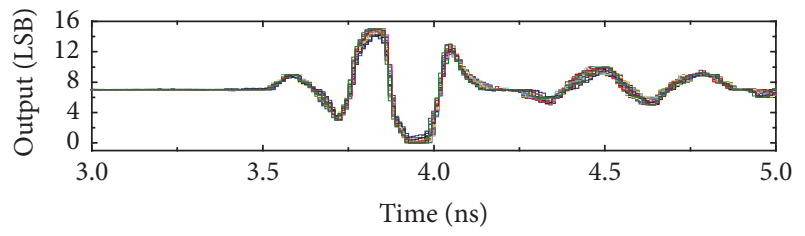

(a)

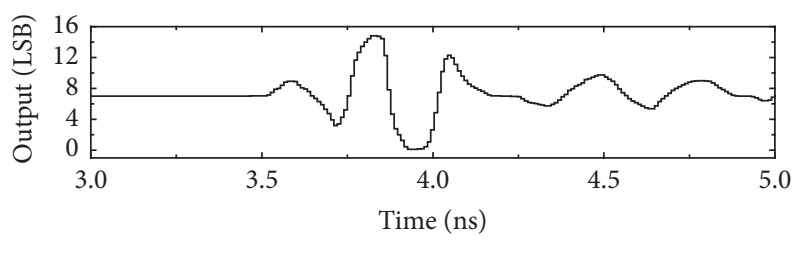

T3R7ref

(b)

FIGURE 13: The aligned signals and the corresponding reference signal for $\mathrm{Tx} 3-\mathrm{Rx} 7$ antenna pair.

the direct wave is almost eliminated and the target response is successfully extracted by both the ideal removal method and the reference method. Theoretically, the direct wave should be absolutely removed by subtracting the without signal. However, because of the sampling error, the waveforms have little variation, resulting in a small residual noise in the differential signal. Meanwhile, since the reference signal is generated by averaging the signals in different position, the random noise is suppressed. Therefore, by using the reference signal, the artifact can be eliminated to the same level as the ideal one in this system.

Figure 16 shows the confocal imaging results employing the signals by different methods. The processing time of the signal and image processing is about 40 seconds using a personal computer with i7-4790 CPU and $8 \mathrm{~GB}$ memory. Figures 16(a) 16(c) show the confocal images using the unaligned signals in $x-y, z-y$, and $z-x$ planes, respectively. Figures $16(\mathrm{~d}) \sim 16(\mathrm{f})$ show the confocal images using the aligned signals in $x-y, z-y$, and $z-x$ planes, respectively. Figures $16(\mathrm{~g}) \sim 16(\mathrm{i})$ show the confocal image using the reference signals in $x-y, z-y$, and $z-x$ planes, respectively. The white rectangle outlines indicate the actual size and position of the embedded target. The significant noise in Figures 16(a) 16(c) is the result of the large residue caused by phase shift. Comparing Figures 16(d) 16(f) and Figures 16(g) 16(i), the target position is successfully estimated, demonstrating the feasibility of the rotation system.

Since the sampling error exists in the system, the target signals always have some high-frequency noise caused by the imperfect subtraction between original signals and reference signal. As shown in Figure 17, compared to the target reflection, the noise resulting from imperfect subtraction has a lot of short-term pulses. The spikes may come from the quantum error in analog-digital conversion and the phase error in the equivalent sampling clock.

In order to suppress the noise, a bandpass filter is applied. The spectrum of the target reflection is shown in Figure 18. The center frequency of the signal is about $4 \mathrm{GHz}$. Therefore, the cutoff frequencies are selected as $1 \mathrm{GHz}$ and $7 \mathrm{GHz}$. The filtered signals are shown in Figure 19. It can be seen that the noise is suppressed and the target reflection become smoother.

The confocal image by use of the signals after the bandpass filter is shown in Figure 20. The white rectangle outlines indicate the actual size and position of the embedded target. 


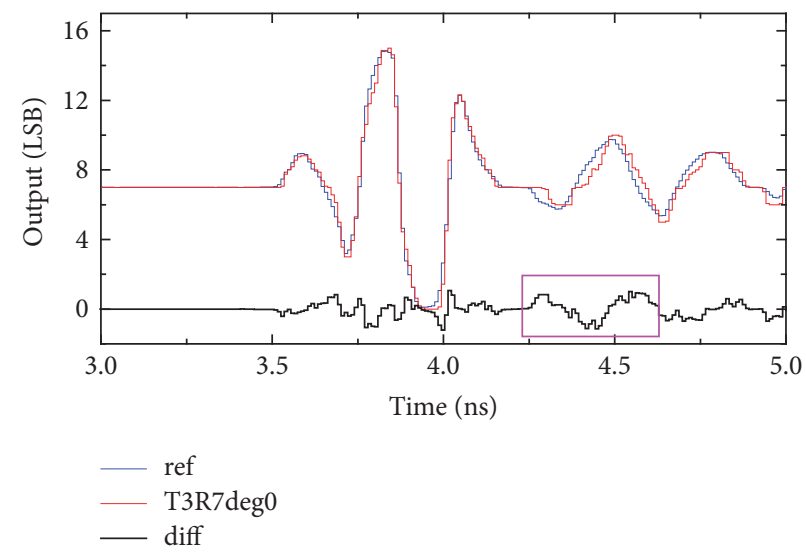

(a)

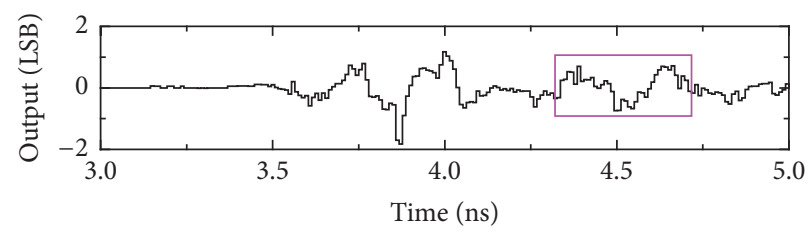

$-60^{\circ}$

(b)

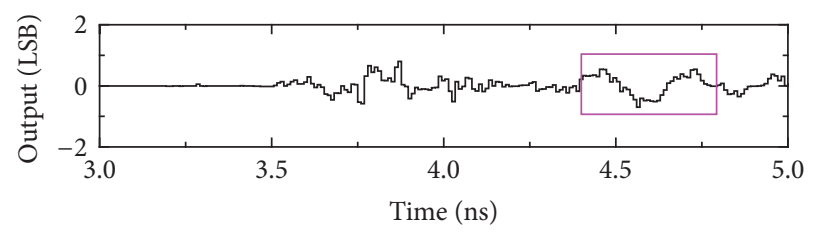

$-120^{\circ}$

(d)

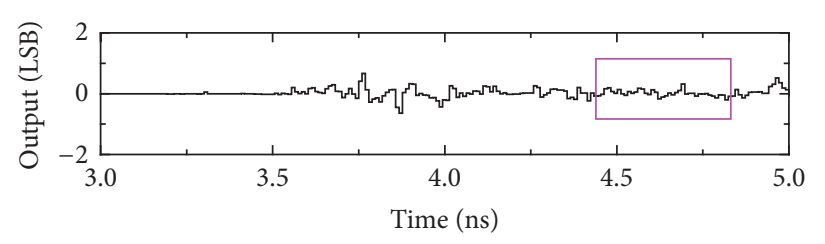

$-180^{\circ}$

(f)

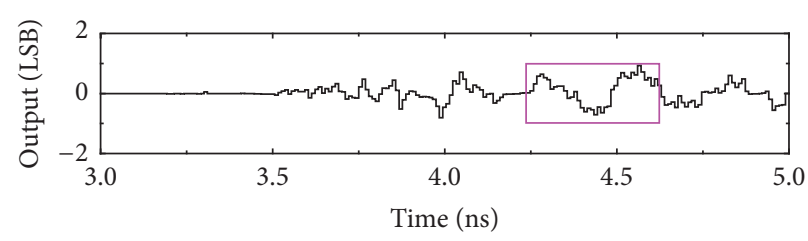

$-300^{\circ}$

(h)

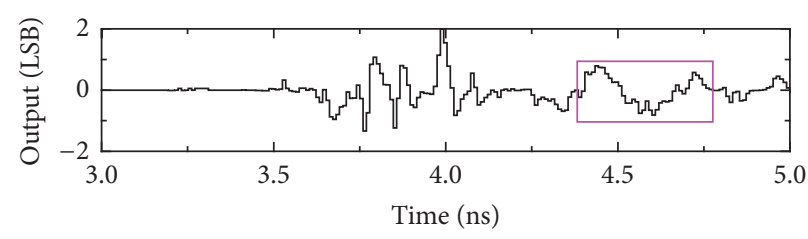

$-90^{\circ}$

(c)

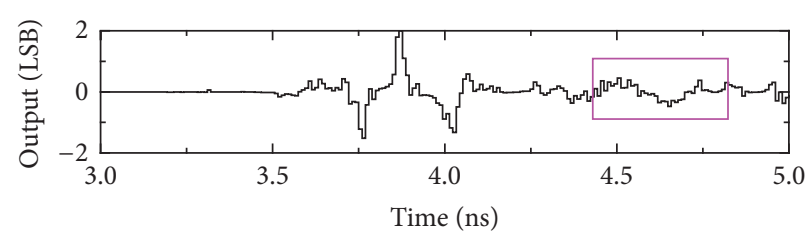

$-160^{\circ}$

(e)

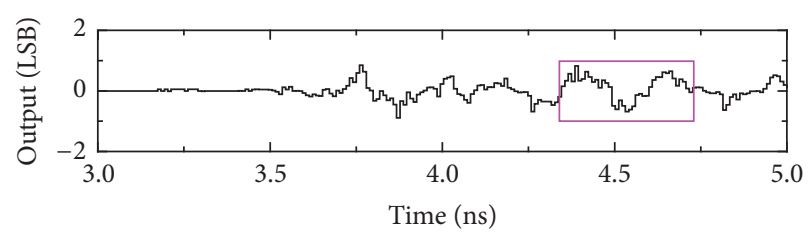

$-250^{\circ}$

(g)

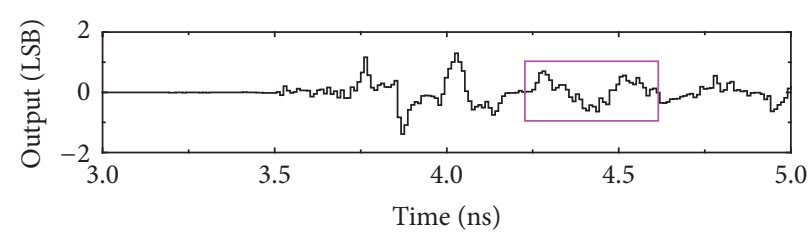

$-330^{\circ}$

(i)

FIGURE 14: The extraction of target reflection and the differential waveforms at different rotation angles. 
TABLE 1: SCR, SMR, and position error of confocal images for different target signal formations.

\begin{tabular}{|c|c|c|c|}
\hline Target signal formation & SCR & SMR & $\begin{array}{l}\text { Position error } \\
(d x, d y, d z) \\
(\mathrm{mm})\end{array}$ \\
\hline Subtracted unaligned signal & -0.89 & 3.74 & $(28,23,5)$ \\
\hline Subtracted aligned signal & 0.71 & 4.22 & $(-2.5,-1.5,1)$ \\
\hline Subtracted reference signal with averaging & 2.24 & 21.15 & $(-2.5,-2,2)$ \\
\hline $\begin{array}{l}\text { Subtracted reference signal with averaging and } \\
\text { filtering }\end{array}$ & 4.94 & 24.61 & $(-2,-2,2)$ \\
\hline
\end{tabular}

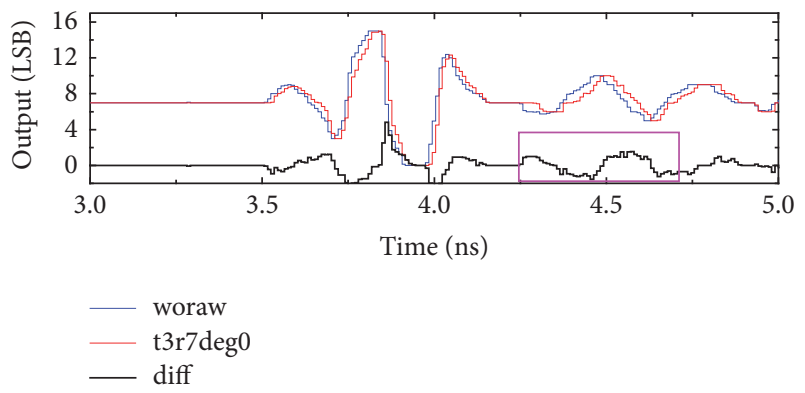

(a)

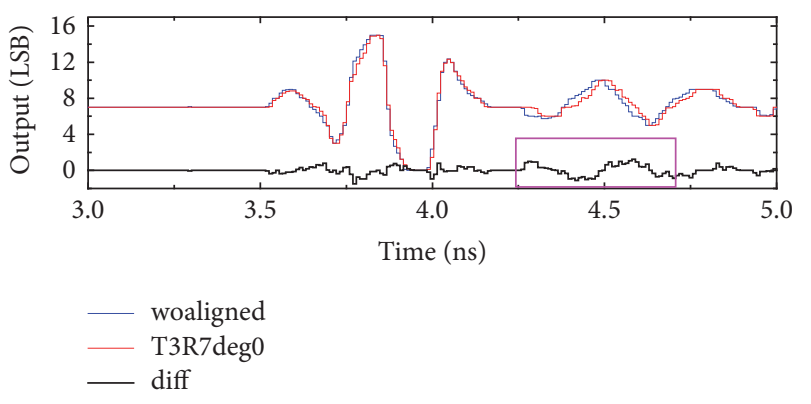

(b)

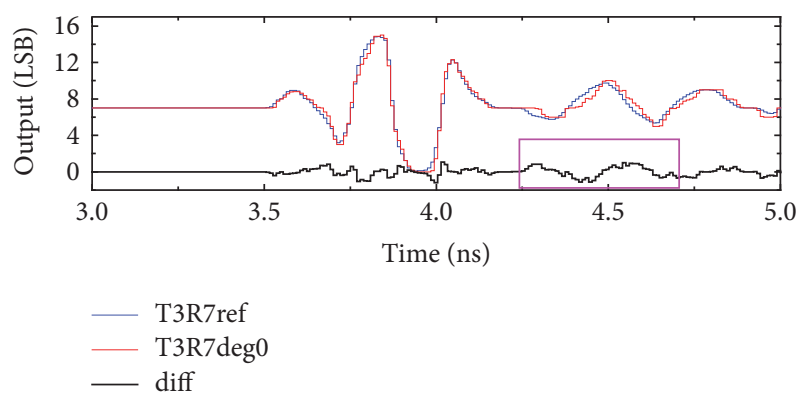

(c)

FIGURE 15: Output waveforms of differential signals. (a) Unaligned signal. (b) Aligned signal. (c) Reference signal with averaging.

Table 1 shows the comparison of signal-to-clutter ratio (SCR), signal-to-mean ratio (SMR), and position error of confocal images for different target signal formations. Without alignment, a large residue was generated by the jitter after subtraction, resulting in the false detection. After phase shift compensation, the SCR was improved and the target was successfully recognized. The SCR was improved by use of the reference signal because the reference signal was averaged by 36 times, so that the random noise was suppressed. After bandpass filtering, the SCR and SMR were improved furthermore. The results demonstrate the effectiveness of the data processing methods.

\section{Conclusion}

A rotating antenna array system was proposed for breast cancer detection using the CMOS integrated circuits. The radar operation using CMOS integrated circuits was demonstrated without any off-the-shelf large equipment. The target signal could be extracted from the differential signal between the received signal and the reference signal which was acquired by averaging the signals at different rotation points. The jitter in this system was suppressed by the phase shift compensation with alignment based on the least square method during the formation of differential signals. The bandpass filter was applied to remove the high-frequency noise which was caused by the imperfect subtraction for differential signals. After alignment and filtering, the highest SCR and SMR were achieved, demonstrating the feasibility of the proposed rotation system and the signal processing methods. Future work includes applying the system to heterogeneous phantoms and optimizing the imaging algorithm for more complex scenarios.

\section{Conflicts of Interest}

The authors declare that there are no conflicts of interest regarding the publication of this paper.

\section{Acknowledgments}

The authors would like to thank Japan Agency for Medical Research and Development (AMED), Grant-in-Aid for Scientific Research, Japan Society of the Promotion of Science (JSPS), and China Scholarship Council (CSC) for their support. 


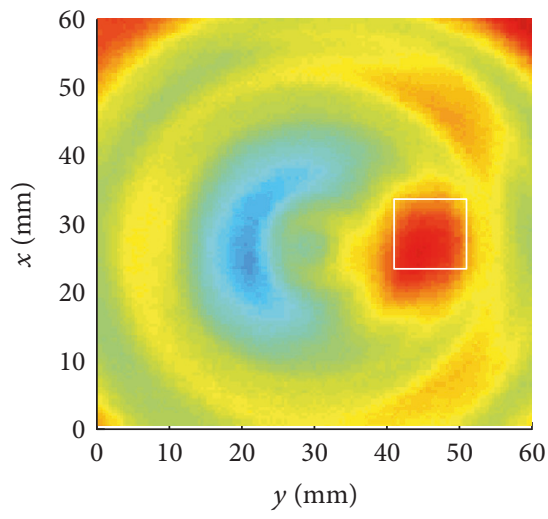

(a)

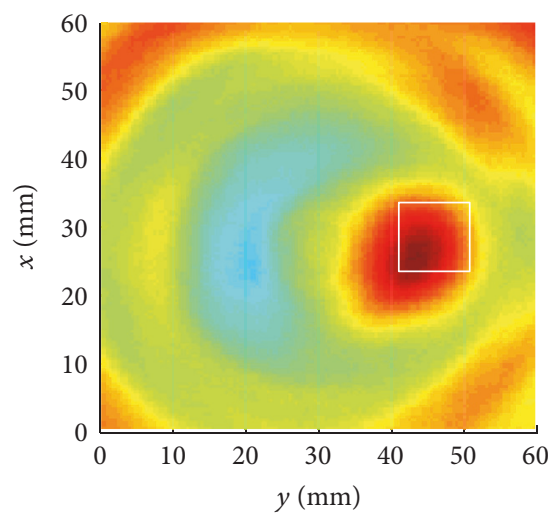

(d)

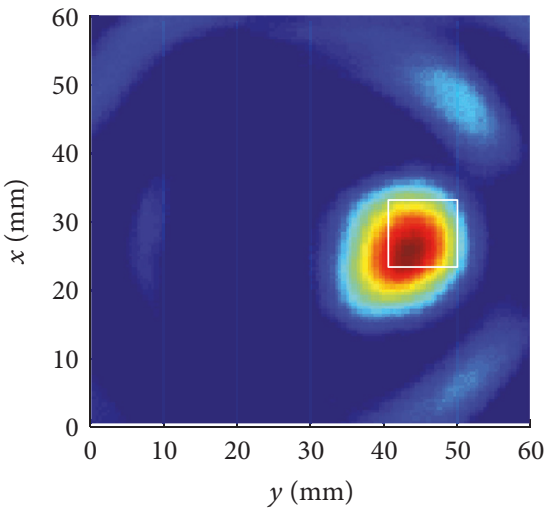

(g)

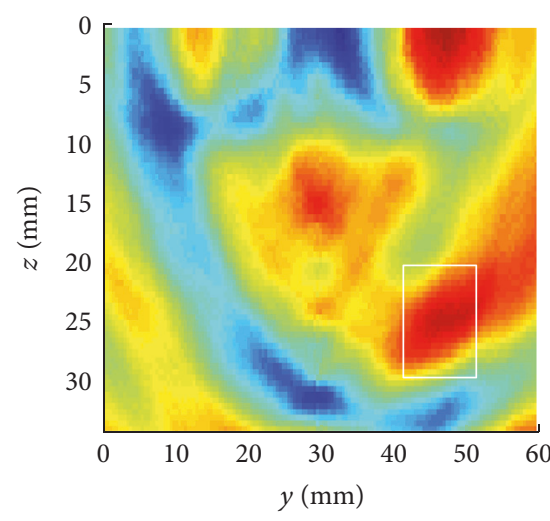

(b)

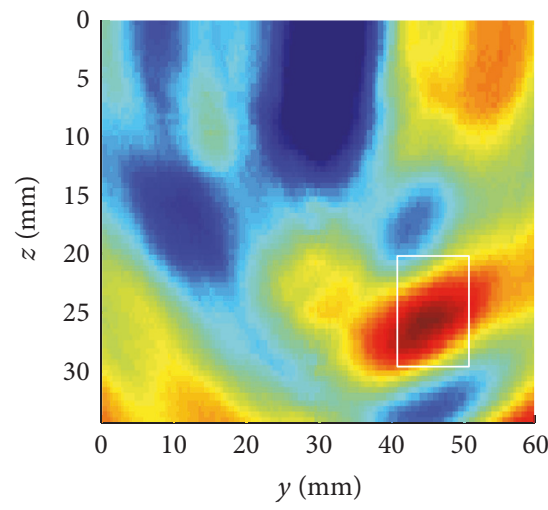

(e)

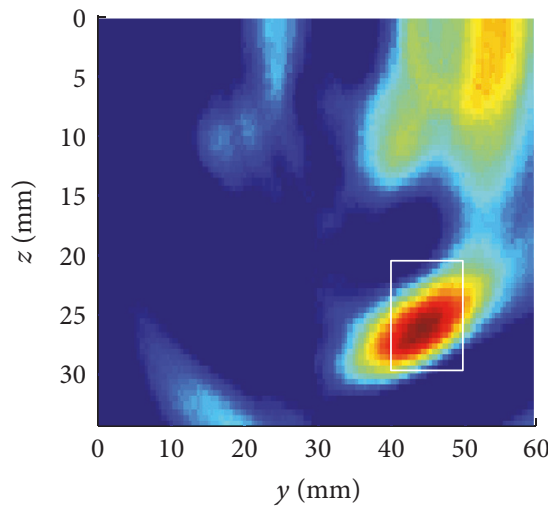

(h)

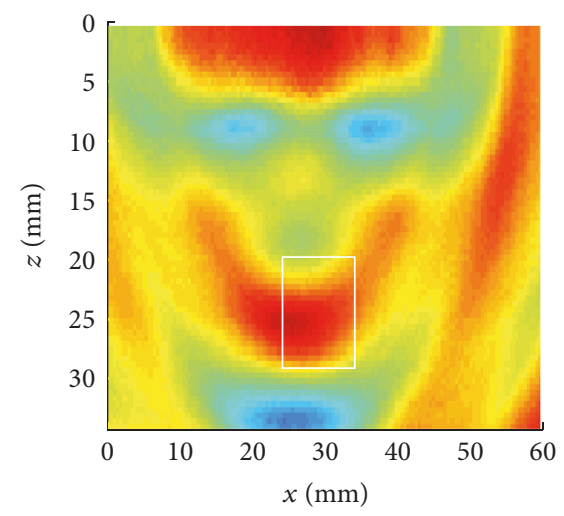

(c)

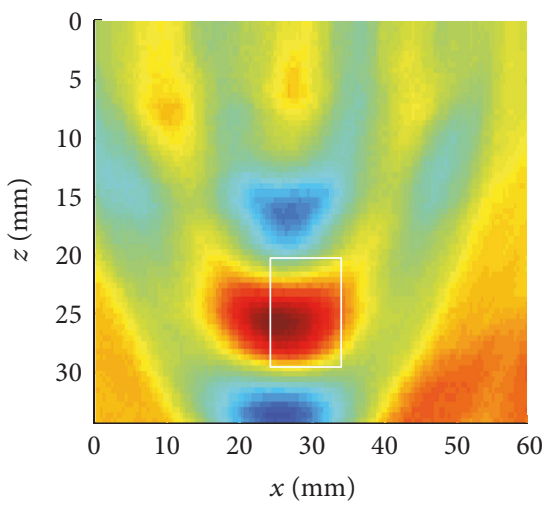

(f)

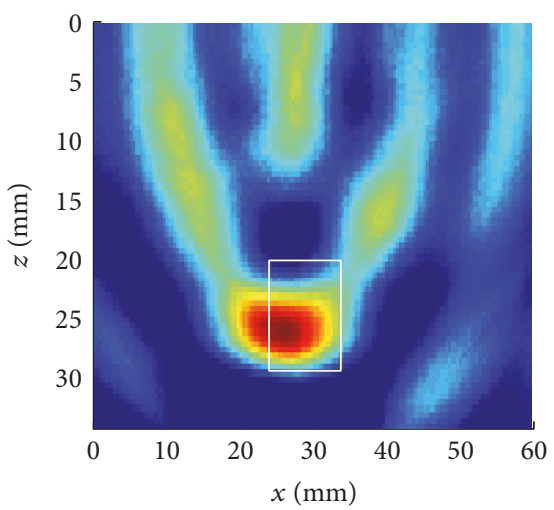

(i)

FIGURE 16: Confocal image results. (a) (c) Unaligned signal. (d) (f) Aligned signal. (g) (i) Reference signal with averaging.

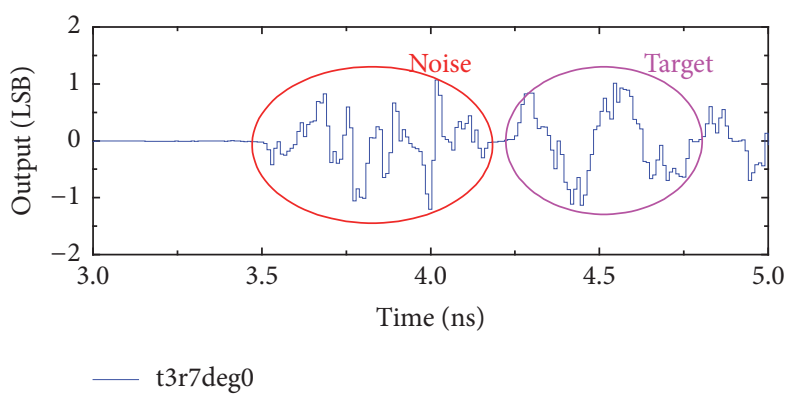

FIGURE 17: The subtracted signal from Tx3-Rx7 antenna pair at 0 degrees. 

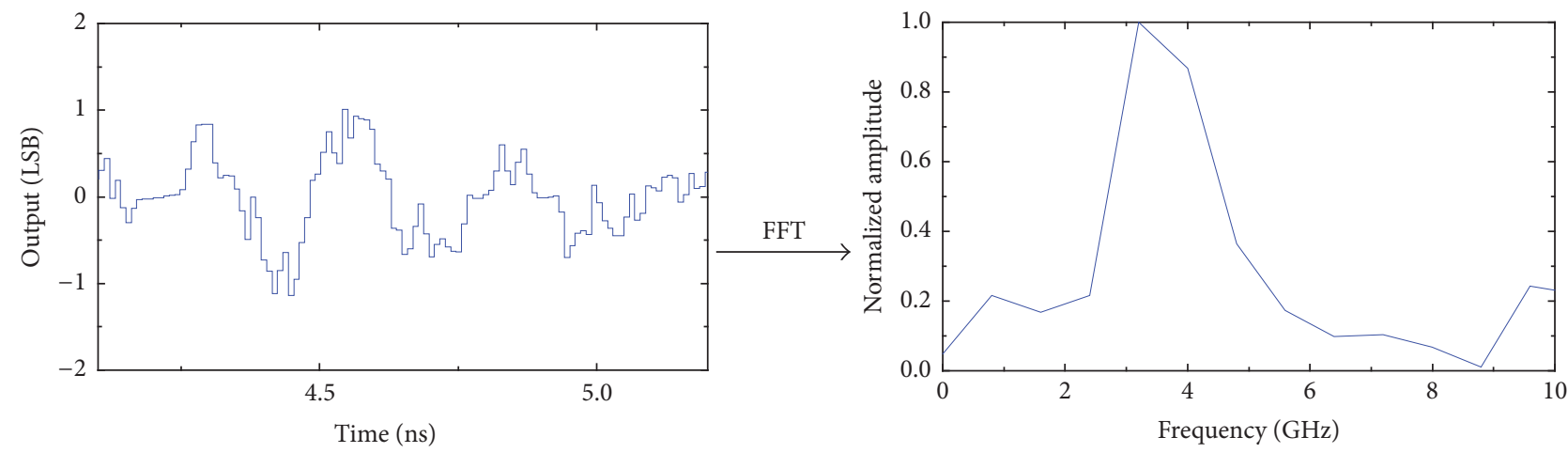

- $\mathrm{t} 3 \mathrm{r} 7 \mathrm{deg} 0$

FIGURE 18: The target reflection in time domain and frequency domain.
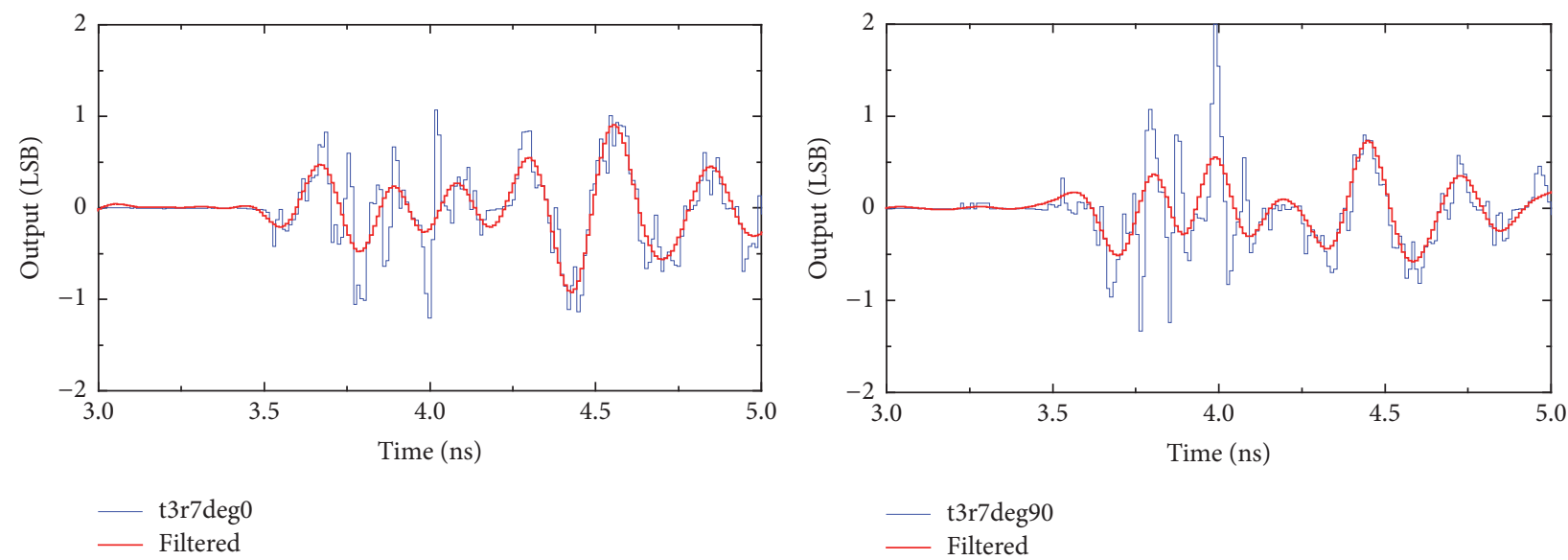

FIGURE 19: The subtracted signals before and after filtering.
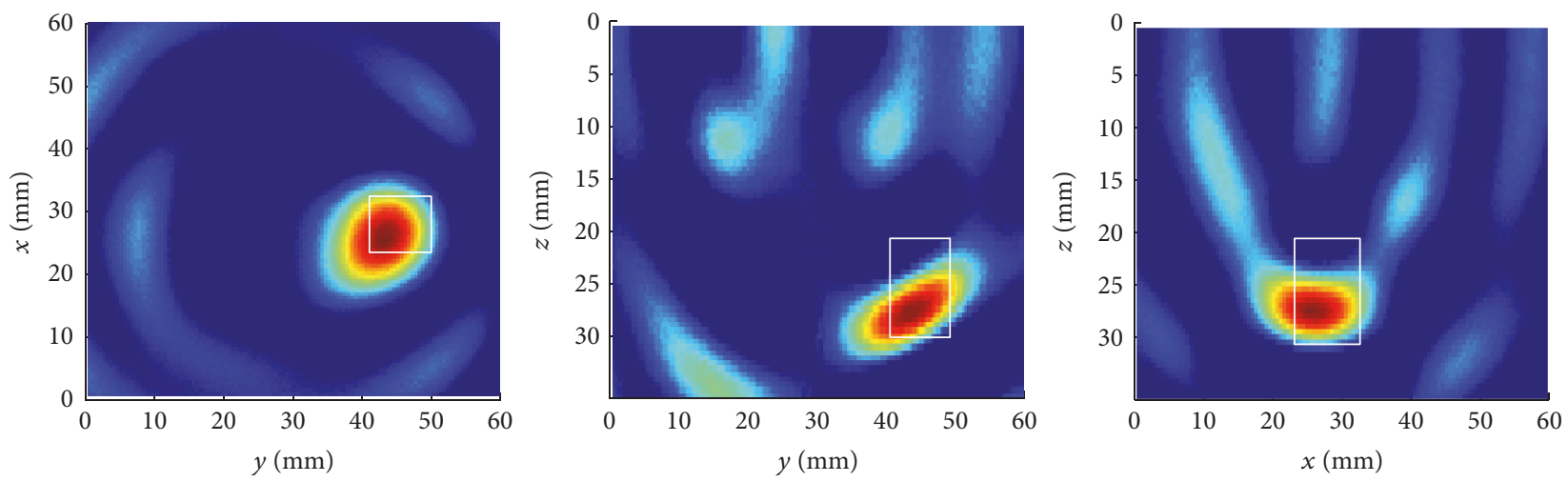

FIGURE 20: Confocal images by use of the filtered target signals.

\section{References}

[1] M. Lazebnik, D. Popovic, L. McCartney et al., "A large-scale study of the ultrawideband microwave dielectric properties of normal, benign and malignant breast tissues obtained from cancer surgeries," Physics in Medicine and Biology, vol. 52, no. 20, pp. 6093-6115, 2007.
[2] M. Lazebnik, L. McCartney, D. Popovic et al., "A large-scale study of the ultrawideband microwave dielectric properties of normal breast tissue obtained from reduction surgeries," Physics in Medicine and Biology, vol. 52, no. 10, article no. 001, 2007.

[3] T. Sugitani, S.-I. Kubota, S.-I. Kuroki et al., "Complex permittivities of breast tumor tissues obtained from cancer surgeries," Applied Physics Letters, vol. 104, no. 25, Article ID 253702, 2014. 
[4] J. D. Shea, P. Kosmas, S. C. Hagness, and B. D. Van Veen, "Three-dimensional microwave imaging of realistic numerical breast phantoms via a multiple-frequency inverse scattering technique," Medical Physics, vol. 37, no. 8, pp. 4210-4226, 2010.

[5] J. D. Shea, B. D. Van Veen, and S. C. Hagness, "A TSVD analysis of microwave inverse scattering for breast imaging," IEEE Transactions on Biomedical Engineering, vol. 59, no. 4, pp. 936-945, 2012.

[6] J. E. Johnson, T. Takenaka, K. A. H. Ping, S. Honda, and T. Tanaka, "Advances in the 3-D forward-backward timestepping (FBTS) inverse scattering technique for breast cancer detection," IEEE Transactions on Biomedical Engineering, vol. 56, no. 9, pp. 2232-2243, 2009.

[7] C. Yu, M. Yuan, J. Stang et al., "Active microwave imaging II: 3-D system prototype and image reconstruction from experimental data," IEEE Transactions on Microwave Theory and Techniques, vol. 56, no. 4, pp. 991-1000, 2008.

[8] Q. Fang, P. M. Meaney, and K. D. Paulsen, "Viable threedimensional medical microwave tomography: theory and numerical experiments," Institute of Electrical and Electronics Engineers. Transactions on Antennas and Propagation, vol. 58, no. 2, pp. 449-458, 2010.

[9] X. Li and S. C. Hagness, "A confocal microwave imaging algorithm for breast cancer detection," IEEE Microwave and Wireless Components Letters, vol. 11, no. 3, pp. 130-132, 2001.

[10] X. Li, S. K. Davis, S. C. Hagness, D. W. Van Der Weide, and B. D. Van Veen, "Microwave imaging via space-time beamforming: Experimental investigation of tumor detection in multilayer breast phantoms," IEEE Transactions on Microwave Theory and Techniques, vol. 52, no. 8, pp. 1856-1865, 2004.

[11] H. Been Lim, N. Thi Tuyet Nhung, E.-P. Li, and N. Duc Thang, "Confocal microwave imaging for breast cancer detection: Delay-multiply-and-sum image reconstruction algorithm," IEEE Transactions on Biomedical Engineering, vol. 55, no. 6, article no. 9, pp. 1697-1704, 2008.

[12] N. R. Epstein, P. M. Meaney, and K. D. Paulsen, "Microwave tomographic imaging utilizing low-profile, rotating, right angle-bent monopole antennas," International Journal of Antennas and Propagation, vol. 2014, Article ID 431602, 2014.

[13] N. R. Epstein, P. M. Meaney, and K. D. Paulsen, "3D paralleldetection microwave tomography for clinical breast imaging," Review of Scientific Instruments, vol. 85, no. 12, Article ID 124704, 2014.

[14] M. Klemm, J. A. Leendertz, D. Gibbins, I. J. Craddock, A. Preece, and R. Benjamin, "Microwave radar-based breast cancer detection: Imaging in inhomogeneous breast phantoms," IEEE Antennas and Wireless Propagation Letters, vol. 8, pp. 1349-1352, 2009.

[15] M. Klemm, J. A. Leendertz, D. Gibbins, I. J. Craddock, A. Preece, and R. Benjamin, "Microwave radar-based differential breast cancer imaging: Imaging in homogeneous breast phantoms and low contrast scenarios," IEEE Transactions on Antennas and Propagation, vol. 58, no. 7, pp. 2337-2344, 2010.

[16] E. Porter, E. Kirshin, A. Santorelli, M. Coates, and M. Popoví, "Time-domain multistatic radar system for microwave breast screening," IEEE Antennas and Wireless Propagation Letters, vol. 12, pp. 229-232, 2013.

[17] E. Porter, A. Santorelli, R. Kazemi, and M. Popović, "Microwave time-domain radar: Healthy tissue variations over the menstrual cycle," IEEE Antennas and Wireless Propagation Letters, vol. 14, pp. 1310-1313, 2015.
[18] E. Porter, M. Coates, and M. Popovic, "An Early Clinical Study of Time-Domain Microwave Radar for Breast Health Monitoring," IEEE Transactions on Biomedical Engineering, vol. 63, no. 3, pp. 530-539, 2016.

[19] E. C. Fear, J. Bourqui, C. Curtis, D. Mew, B. Docktor, and C. Romano, "Microwave breast imaging with a monostatic radar-based system: A study of application to patients," IEEE Transactions on Microwave Theory and Techniques, vol. 61, no. 5, pp. 2119-2128, 2013.

[20] J. Bourqui, J. M. Sill, and E. C. Fear, "A prototype system for measuring microwave frequency reflections from the breast," International Journal of Biomedical Imaging, vol. 2012, Article ID 851234, 2012.

[21] A. T. Mobashsher, A. Mahmoud, and A. M. Abbosh, "Portable Wideband Microwave Imaging System for Intracranial Hemorrhage Detection Using Improved Back-projection Algorithm with Model of Effective Head Permittivity," Scientific Reports, vol. 6, Article ID 20459, 2016.

[22] M. Bassi, M. Caruso, M. S. Khan, A. Bevilacqua, A.-D. Capobianco, and A. Neviani, "An integrated microwave imaging radar with planar antennas for breast cancer detection," IEEE Transactions on Microwave Theory and Techniques, vol. 61, no. 5, pp. 2108-2118, 2013.

[23] A. Santorelli, E. Porter, E. Kang, T. Piske, M. Popović, and J. D. Schwartz, "A Time-Domain Microwave System for Breast Cancer Detection Using a Flexible Circuit Board," IEEE Transactions on Instrumentation and Measurement, vol. 64, no. 11, pp. 29862994, 2015.

[24] T. Kikkawa, P. K. Saha, N. Sasaki, and K. Kimoto, "Gaussian monocycle pulse transmitter using $0.18 \mu \mathrm{m}$ CMOS technology with on-chip integrated antennas for inter-chip UWB communication," IEEE Journal of Solid-State Circuits, vol. 43, no. 5, pp. 1303-1311, 2008.

[25] N. Sasaki, K. Kimoto, W. Moriyama, and T. Kikkawa, "A singlechip ultra-wideband receiver with silicon integrated antennas for inter-chip wireless interconnection," IEEE Journal of SolidState Circuits, vol. 44, no. 2, pp. 382-393, 2009.

[26] A. Azhari, S. Takumi, S. Kenta, T. Kikkawa, and X. Xiao, "A $17 \mathrm{GHz}$ bandwidth $1.2 \mathrm{~mW}$ CMOS switching matrix for UWB breast cancer imaging," in 10th IEEE Biomedical Circuits and Systems Conference, BioCAS 2014, pp. 109-112, che, October 2014.

[27] A. Toya, K. Sogo, N. Sasaki, and T. Kikkawa, "125mW 102.4 GS/s ultra-high-speed sampling circuit for complementary metaloxide-semiconductor breast cancer detection system," Japanese Journal of Applied Physics, vol. 52, no. 4, Article ID 04CE07, 2013.

[28] A. Toya, N. Sasaki, S. Kubota, and T. Kikkawa, "Confocal Imaging System Using High-Speed Sampling Circuit and UltraWideband Slot Antenna," Japanese Journal of Applied Physics, vol. 50, no. 4S, p. 04DE02, 2011.

[29] S. Kubota, A. Toya, T. Sugitani, and T. Kikkawa, "5-Gb/s and 10$\mathrm{GHz}$ center-frequency gaussian monocycle pulse transmission using 65-nm logic CMOS with on-chip dipole antenna and high- $\kappa$ interposer," IEEE Transactions on Components, Packaging and Manufacturing Technology, vol. 4, no. 7, pp. 1193-1200, 2014.

[30] T. Sugitani, S. Kubota, M. Hafiz, X. Xiao, and T. Kikkawa, "Three-dimensional Confocal imaging for breast cancer detection using CMOS Gaussian monocycle pulse transmitter and $4 \times 4$ ultra wideband antenna array with impedance matching layer," Japanese Journal of Applied Physics, vol. 53, no. 4, Article ID 04EL03, 2014. 
[31] H. Song, H. Kono, Y. Seo et al., "A Radar-Based Breast Cancer Detection System Using CMOS Integrated Circuits," IEEE Access, vol. 3, pp. 2111-2121, 2015.

[32] T. Sugitani, S. Kubota, A. Toya, X. Xiao, and T. Kikkawa, "A Compact $4 \times 4$ Planar UWB Antenna Array for 3-D Breast Cancer Detection," IEEE Antennas and Wireless Propagation Letters, vol. 12, pp. 733-736, 2013. 


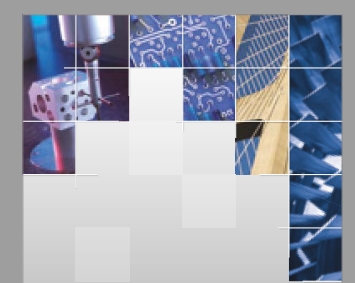

\section{Enfincering}
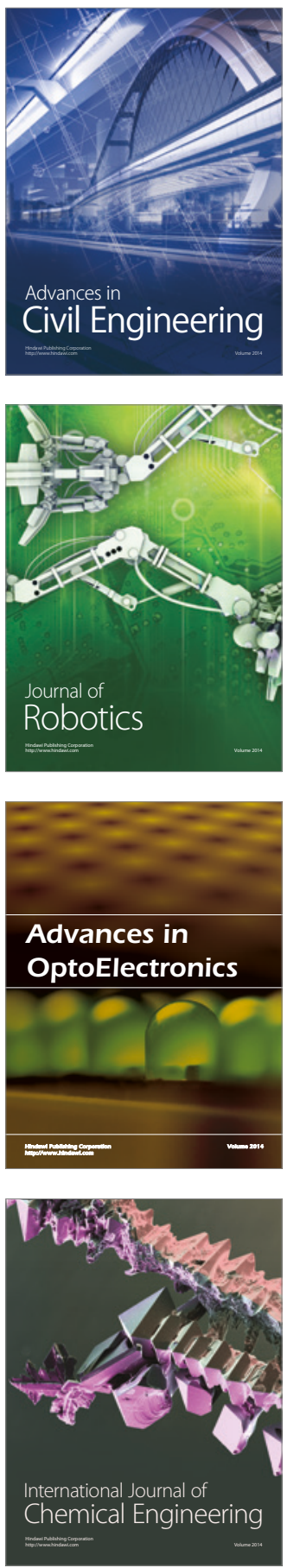

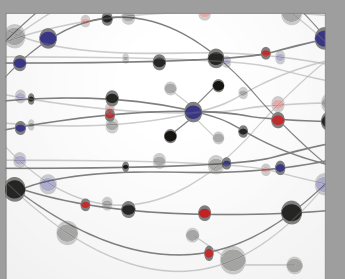

The Scientific World Journal

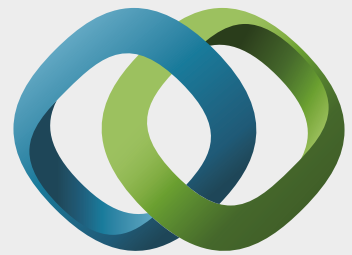

\section{Hindawi}

Submit your manuscripts at

https://www.hindawi.com
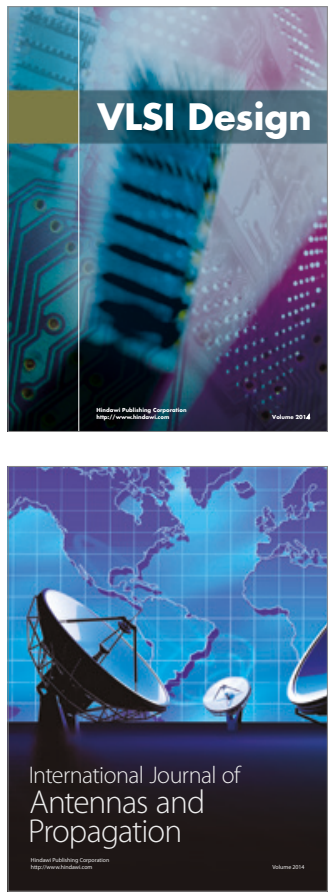

\section{Rotating}

Machinery
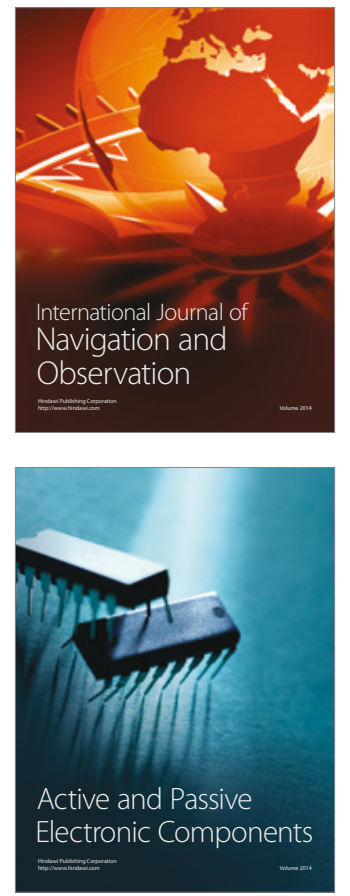
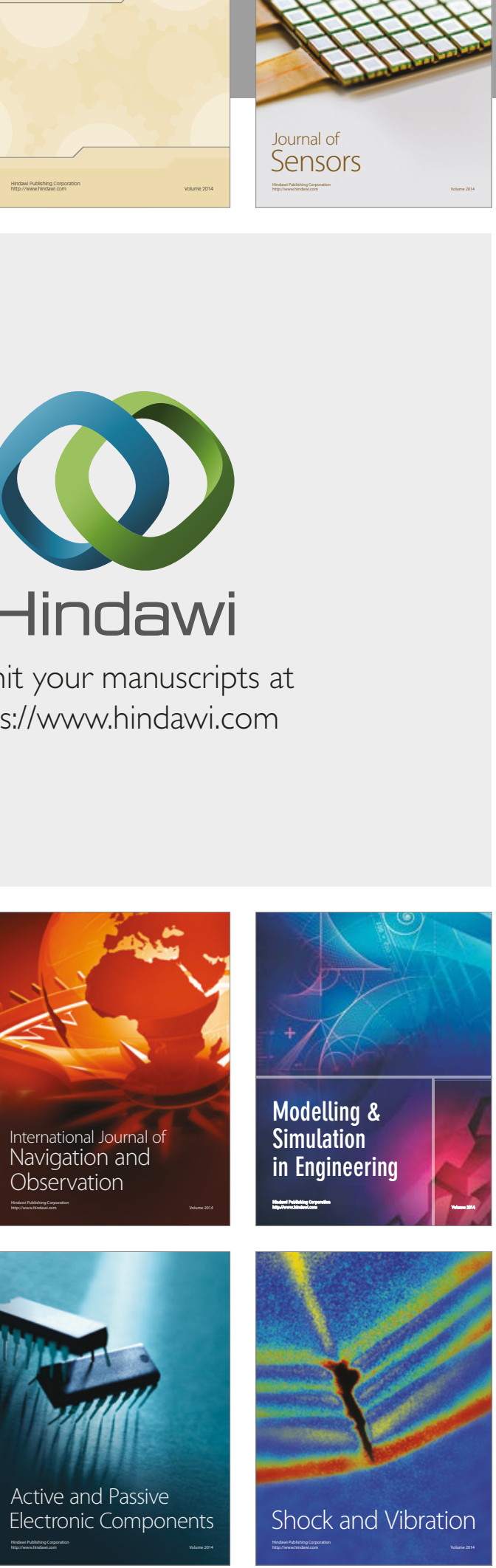
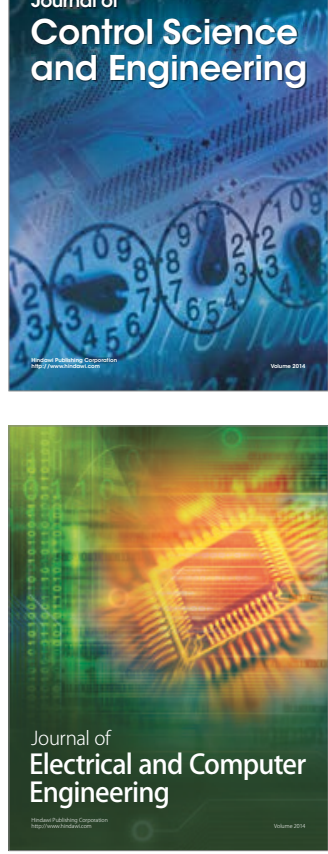

Distributed

Journal of

Control Science

and Engineering
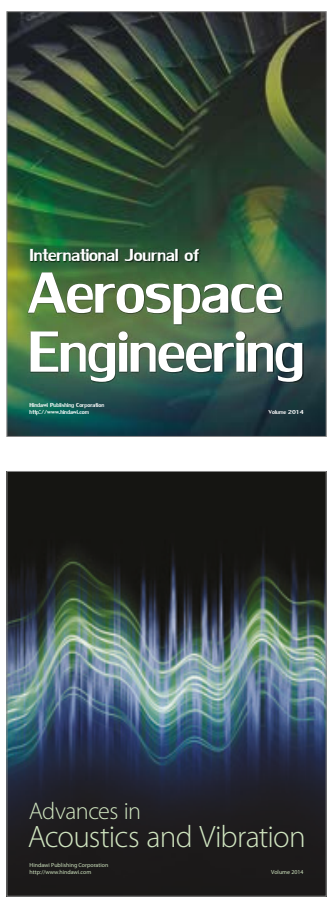

Sensor Networks 